\title{
Connectivity and Mobility Aware Dynamic Clustering in VANETs
}

\author{
M. S. Kakkasageri and S. S. Manvi
}

\begin{abstract}
In this position paper, we present dynamic and stable clustering scheme in Vehicular Ad-Hoc Networks (VANET) based on relative speed and connectivity among neighboring vehicles. The proposed scheme uses multiagent system to form dynamic and stable clusters in VANETs on a lane between two intersections by considering vehicle speed, direction, connectivity degree to other vehicles and mobility pattern. Multiagent system comprises of heavy-weight static and light-weight mobile agents. Clustering performance is measured in terms of cluster formation time and cluster head selection time.
\end{abstract}

Index Terms-VANETs, multiagents, dynamic clusters.

\section{INTRODUCTION}

Clustering can be used in VANETs to partition the network into smaller groups of moving vehicles embedded with computing and networked devices. This has several benefits including efficient usage of bandwidth, distribution of resources and scalability [1]. The clustering in VANETs has to consider the following unique features of network: vehicle movement constrained by roads, road side units (RSUs) such as signs and traffic lights leading vehicles to follow some traffic patterns, vehicles are equipped with Global Positioning Systems (GPS) devices providing knowledge about geographical position, distributed operation, topology variations due to mobility and mobility pattern. Cluster formation plays a key role in VANETs for information gathering, aggregation and dissemination. Dynamic clustering is a technique to form stable grouping of computing devices on the fly that does not have physical connections.

Some of the challenges to be considered for dynamic clustering in VANETs are vehicle mobility, direction, relative speed and mobility pattern. Objectives of forming dynamic clusters are as follows: moving cluster formation in minimum time, maintenance of moving cluster with reduced overheads and cluster reconfiguration due to cluster head change because of mobility.

Several works have been reported in VANETs dealing with mobility pattern of vehicles and formation of clusters. Study of VANET characteristics and different mobility patterns of vehicles is presented in [2]. A car-following model using a neural network approach for mapping perceptions to actions is developed in [3]. The model has a

Manuscript received December 29, 2012; revised April 12, 2013.

M. S. Kakkasageri is with the E \& C Engineering Department, Basaveshwar Engineering College, Bagalkot-587102, Karnataka, India (e-mail: mahabalesh sk@yahoo.co.in).

S. S. Manvi is with the E \& C Engineering Department, Reva Institute of Technology and Management, Bangalore-560064, Karnataka, India (e-mail: sunil.manvi@revainstitution.org). similar formulation to the desired spacing models that does not consider reaction time and attempt to explain the behavioral aspects of the following car.

An inter vehicle information dissemination protocol called received message-dependent protocol (RMDP) that propagates the preceding traffic information to the following vehicles is discussed in [4]. RMDP protocol autonomously changes the dissemination interval depending on number of reception messages and detected reception errors in order to avoid message collision among vehicles. Using RMDP, many vehicles can acquire preceding traffic information within short periods under light and heavy traffic conditions.

Mobility-based stable clustering scheme in VANETs which utilizes the affinity propagation algorithm in a distributed manner is presented in [5]. Vehicular mobility during cluster formation is considered in affinity propagation algorithm. In Affinity Propagation for Vehicular networks (APROVE), each node in the network transmits the responsibility and availability messages to its neighbors and then makes an independent decision on clustering. This results, in a distributed algorithm where every node is clustering with the nodes in its one-hop neighborhood.

The proposed multiagent based dynamic clustering scheme consists of heavy-weight static and light-weight mobile agents that consider the parameters vehicle speed and stable connectivity. It consists of two phases: identification of cluster members and dynamic cluster formation with cluster head selection.

The rest of the paper is organized as follows. Section II presents proposed multiagent based dynamic clustering in VANETs. Simulation model for proposed scheme and result analysis is presented in section III. Finally, section IV concludes the work and briefs future work.

\section{Multiagent Based DynamiC Clustering}

In this section, we briefly describe about software agents. Network environment considered for the proposed work, description of the clustering agency components and dynamic clustering scheme using the agency are also presented in this section.

\section{A. Software Agents}

Agents are the autonomous programs which sense the environment and acts upon the environment using its knowledge to achieve their goals [6]-[9]. The agent environment generally referred as a host system, network, a user via a graphical user interface, a collection of other agents, or perhaps all of these combined. Agents are classified as single agent and multiagent systems (MAS). Single agent systems comprise of a single agent interacting with resources, 
humans and other processes to perform a dedicated task. MAS comprises of set of agents that interact, cooperate, and coordinate with each other to perform a set of tasks or a dedicated tasks. Mobile agents are multiagent systems, which roam in the network to achieve their goals [10].

\section{B. Network Environment}

We consider a VANET in which numbers of vehicles are separated by certain distance (between consecutive vehicles). The VANET is purely based on vehicle-to-vehicle (V2V) architecture. We assume that vehicles move on a road as shown in Fig. 1. All vehicles are equipped with GPS. Each vehicle is loaded with a location digital map and is concerned about road information ahead of it on its way to forward direction. A lane segment ends at an intersection. Each vehicle is equipped with an agent platform to support the proposed agency.

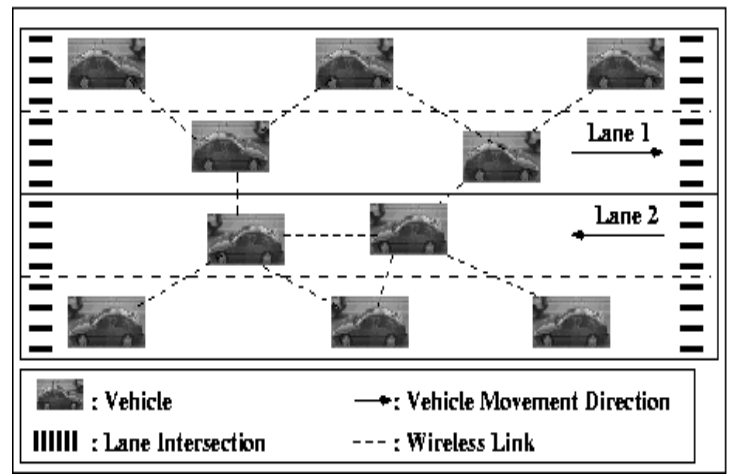

Fig. 1. VANET Environment.

\section{Dynamic Clustering Agency}

This section describes the dynamic clustering agency located in a vehicle that is a part of VANET. Dynamic clustering agency takes the comprehensive decisions on selection of cluster members and cluster head. The components and interactions of the agency are depicted in Fig. 2.

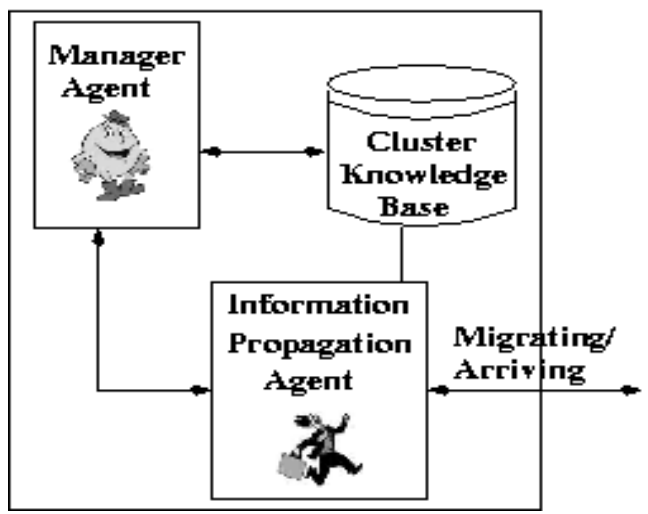

Fig. 2. Dynamic Clustering Agency.

It consists of a manager agent (MA) and information propagation agent (IPA). MA is a static agent, where as IPA is mobile agent. The agency also consists of a Cluster Knowledge base (CKB) that works on the principle of blackboard architecture, which is used for inter-agent communication.

- Manager Agent (MA): MA runs on each vehicle, creates IPA and $\mathrm{CKB}$, controls and coordinates dynamic clustering agency. Periodically MA measures the speed of a vehicle and connectivity among neighbors. MA also measures its location using the position information embedded in the vehicle.

- Information Propagation Agent (IPA): IPA is used to form and maintain a dynamic cluster. It distributes the cluster member and cluster head status to all cluster members.

- Cluster Knowledge Base (CKB): It comprises of information of itself and neighboring vehicles. The information about itself are as follows: status of the vehicle as cluster member or cluster head, movements made in recent interval, bandwidth utilized for clustering and mobility pattern. The information about the neighbor is like neighbor IDs and their status and mobility pattern. The knowledge base is read as well as updated by the agents.

\section{Dynamic Clustering}

Functioning of the proposed multiagent based dynamic clustering scheme in VANETs is explained in the following two phases: (1) identification of cluster members and (2) dynamic cluster formation with cluster head selection.

Cluster member identification: The relative speed difference among neighboring vehicles is the prime parameter for identifying cluster members. Vehicles broadcast their speed to all other vehicles within their communication range. Vehicles are said to be neighbors if the distance between them is less than communication range. But all neighboring vehicles may not be suitable for cluster members for a lane due to direction of movement and relative speed. MA selects cluster members among neighbor vehicles by eliminating vehicles traveling in the opposite direction as well vehicles moving with high relative speed in the same direction.

Dynamic cluster formation with cluster head selection: MA of the initiator vehicle triggers IPA to its neighbor cluster members. IPA interacts with the MA of each visited cluster member to confirm its connectivity to the cluster and collects details such as vehicle ID, location and connected vehicles. IPA sends this information to its MA. MA uses the knowledge base to develop the cluster information table for identification of the cluster head. In this way, the dynamic cluster is formed.

Fig. 3 shows an example scenario of dynamic clusters formation process, where two dynamic clusters are formed. Dynamic clusters 1 and 2 consist of three vehicles (V1, V2 and V3) and four vehicles (V4, V5, V6 and V7) respectively.

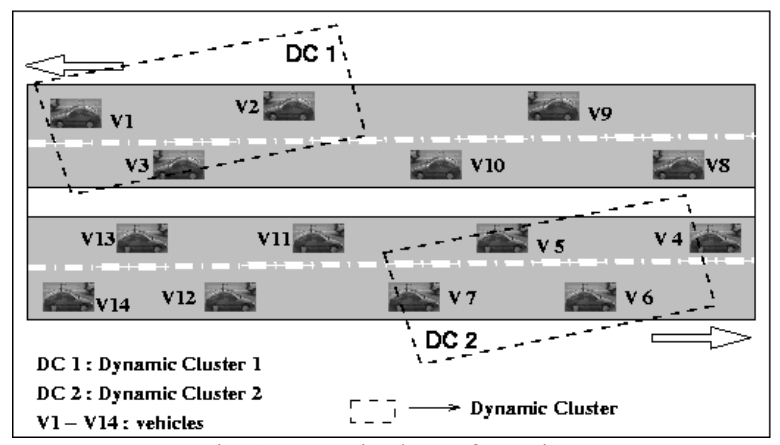

Fig. 3. Dynamic cluster formation. 
MA of the initiator vehicle determines cluster head. The criteria of vehicle's suitability as cluster head is to increase the stability of the cluster structure and maximize its lifetime. Hence, an elected cluster head is expected to stay connected with its members for the longest period of time. Therefore a vehicle connected with more vehicles and less average speed is qualified for winning the cluster head status. Selected cluster head is announced by MA of the initiator vehicle to all the cluster members by triggering IPA.

\section{SimUlATION}

The proposed scheme has been simulated in various network scenarios using " $\mathrm{C}$ " programming language with a confidence interval of $95 \%$. We consider $n$ number of vehicles moving in a fixed region of length $A \mathrm{Km}$. and breadth $B \mathrm{Km}$. We consider vehicles to move in a lane of type $R_{\text {type }}$, freeway with number of $L$ lanes. Communication coverage area for each vehicle is considered as a $V_{\text {com }}$ meters. Coverage area around each vehicle has a bandwidth $B W$ shared among neighbors. It is assumed that there is a free flow movement of vehicles. Safety distance of $R_{s}$ meters is maintained from preceding vehicle for a certain tolerance time and then change lane if possible. Mobility factor for each node is in between the range of $I$ to $J \mathrm{Kmph}$ (Kilometers per hour).

\section{A. Simulation Inputs}

The simulation input parameters considered are as follows: $n=10$ to $50, A=5000 \mathrm{mts}$., $B=30 \mathrm{mts}$., $R_{\text {type }}=$ Free way, $L=$ 2, $V_{\text {com }}=250 \mathrm{mts}$., and $500 \mathrm{mts}$., $B W=5000 \mathrm{Mbps}, R_{s}=4$ mts., $I=40 \mathrm{Kmph}, J=80 \mathrm{Kmph}$.

\section{B. Performance Metrics}

Some of performance metrics evaluated are cluster formation time and cluster head selection time.

- Cluster formation time: It is defined as the time taken by all cluster members to form a dynamic cluster. It is expressed in terms of seconds.

- Cluster head selection time: The total time taken by all cluster members to elect the cluster head. It is expressed in terms of seconds.

\section{Result Analysis}

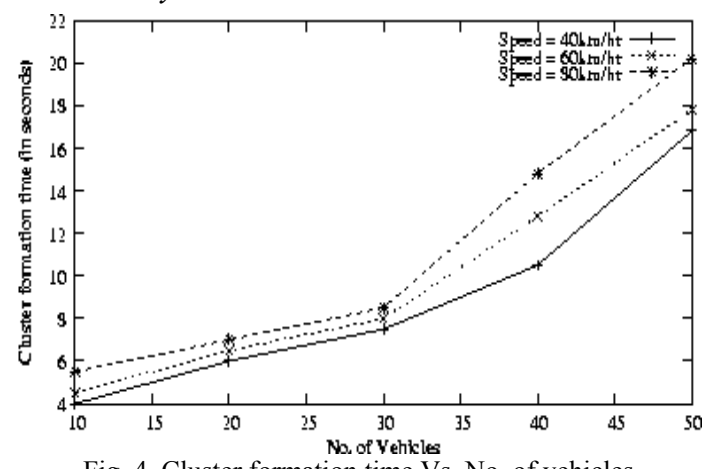

Fig. 4. Cluster formation time Vs. No. of vehicles (Comm. range $=250 \mathrm{mts}$.)

Fig. 4 depicts the comparison of cluster formation time dynamic clustering scheme. For the communication range of a vehicle as $250 \mathrm{mts}$., as the speed of vehicles (from 40 to 80 $\mathrm{Kmph}$ ) and number of vehicles increase, cluster formation time is increased. Cluster formation time is initially low for lower number of vehicles (upto 30) and increases linearly as the number of vehicles increase. This behavior results from the network connectivity in the VANET.

Furthermore, a set of simulations were conducted for the communication range of vehicle as $500 \mathrm{mts}$., as shown in Fig. 5. For different mobility values like $40 \mathrm{Kmph}, 60 \mathrm{Kmph}$ and $80 \mathrm{Kmph}$; as the number of vehicles increase, the performance of the scheme is better than vehicles having communication range $250 \mathrm{mts}$.

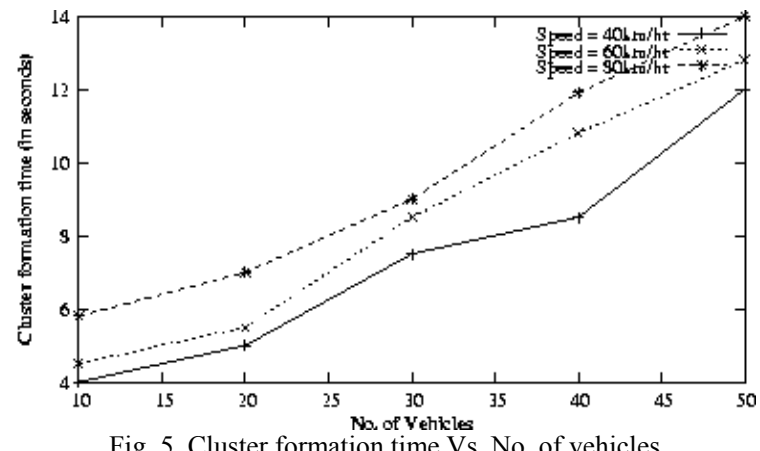

Fig. 5. Cluster formation time Vs. No. of vehicles (Comm. range $=500 \mathrm{mts}$. $)$.

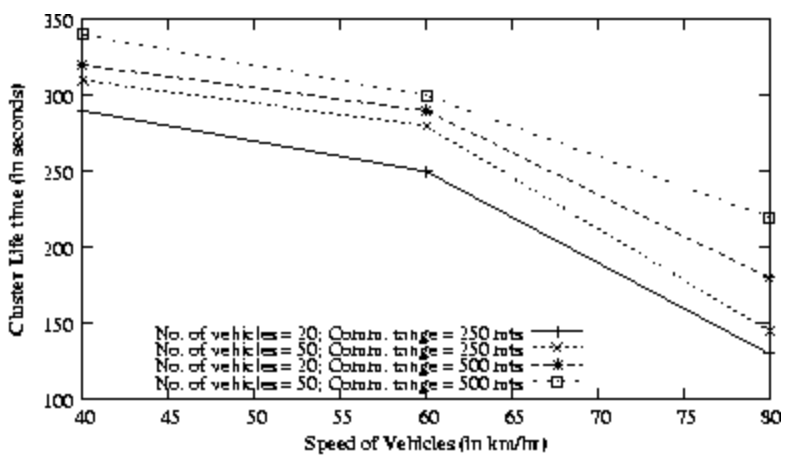

Fig. 6. Cluster life time Vs. Speed of vehicles (in $\mathrm{km} / \mathrm{hr}$ ).

Cluster life time for different speed values (40, 60 and 80 $\mathrm{Kmph}$ ) is illustrated in Fig. 6. Cluster life time decreases as speed of vehicle increases. Cluster life time is relatively high for more number of vehicles with increase in communication range.

\section{CONCLUSION}

In this position paper, we have developed a multiagent based dynamic clustering scheme in VANETs. The scheme forms a moving stable cluster on a lane between two intersections by considering parameters such as vehicle speed, direction and connectivity to other vehicles. Simulation results showed that the proposed scheme performs better in terms of cluster formation time and cluster life time. Further we have planned to analyze some of the performance parameters like cluster member selection time, cluster head selection time and control overheads involved for varying number of vehicles with different mobility values.

\section{REFERENCES}

[1] R. T. Goonewardene, F. H. Ali, and E. Stipidis, "Robust mobility adaptive clustering scheme with support for geographic routing for vehicular ad hoc networks," IET Intelligent Transportation Systems, vol. 3, no. 2, pp. 148-158, 2009 
[2] E. Schoch, F. Kargl, and M. Weber, "Communication Patterns in VANETs," IEEE Communications Magazine, pp. 119-125, November 2008.

[3] S. Panwai and H. Dia, "Neural Agent Car-Following Models," IEEE Transactions on intelligent transportation systems, vol. 8, no. 1, pp. 60-70, March 2007.

[4] M. Saito, J. Tsukamoto, T. Umedu, and T. Higashino, "Design and Evaluation of Inter vehicle Dissemination Protocol for Propagation of Preceding Traffic Information," IEEE Transactions on Intelligent transportation systems, vol. 8, no. 3, pp. 379-390, September 2007.

[5] C. Shea, B. Hassanabadi, and S. Valaee, "Mobility-Based Clustering in VANETs using Affinity Propagation," IEEE Globecom, 2009.

[6] S. Franklin and A. Graser. (1996). Is it an Agent or Just a Program: A Taxonomy for Autonomous Agents. [Online]. Available: http://citeseer.nj.nec.com/32780.html

[7] N. Jennings, "Developing Agent-Based Systems," IEE software engineering, vol. 44, no. 1, pp. 1-2, Feb. 1997.

[8] J. Bradshaw, Software Agents, AAAI Press, California, 2000.

[9] S. Russell and P. Norvig, Artificial Intelligence a Modern Approach, prentice hall, India, 2001

[10] S. S. Manvi and P. Venkataram, "Applications of agent technology in communications: A review," Computer communications, vol. 27, pp. 1493-1508, Oct. 2004.

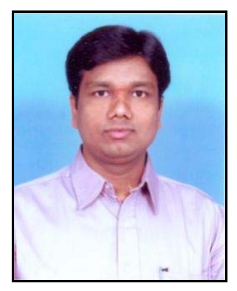

Mahabaleshwar S. Kakkasageri completed his M.Tech and $\mathrm{PhD}$ from Visvesvaraya Technological University Belgaum India. Presently, he is working as
Faculty in Department of Electronics and Communication Engineering, Basaveshwar Engineering College, Bagalkot, Karnataka, India. He has published 20 national and international conference papers and 9 national and international journals. He has also coauthored a book on Wireless and Mobile Networks: Concepts and Protocols, published by Wiley-India. His area of research interest is a wireless network, especially vehicular ad hoc networks, and sensor networks. He is a member of IEEE USA and IETE India.

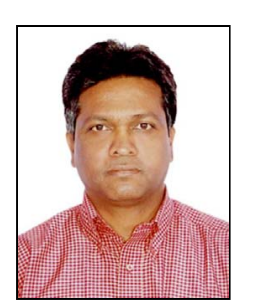

Sunilkumar S. Manvi received M.E. degree in Electronics from the University of Visvesvaraya College of Engineering, Bangalore and Ph.D degree in Electrical Communication Engineering, Indian Institute of Science, Bangalore, India. He is currently working as Dean $(\mathrm{R} \& \mathrm{D})$, Professor and Head of Department of Electronics and Communication Engineering, Reva Institute of Technology and Management, Bangalore, India. He is involved in research of Agent based applications in Multimedia Communications, Grid computing, Vehicular Ad-hoc networks, E-commerce and Mobile computing. He has published about 130 papers in national and international conferences and 80 papers in national and international journals. He has published 3 books. He is a Fellow IETE (FIETE, India), Fellow IE (FIE, India) and member ISTE (MISTE, India), senior member of IEEE (SMIEEE, USA), He has been listed in Marqui's Whos Who in the World. 